\title{
Trastornos de la percepción musical
}

\author{
Musical perception disorders
}

\author{
Bárbara Wipe $\mathbf{U}^{1,3}$, Maya Kuroiwa $\mathbf{R}^{1}$, Paul H. Délano $\mathbf{R}^{2,3}$.
}

\begin{abstract}
RESUMEN
La percepción musical es una experiencia que no sólo involucra componentes cognitivos, sino también respuestas emocionales acorde a la experiencia y cultura del sujeto. El procesamiento musical se inicia a nivel periférico en el sistema auditivo, y a nivel central se divide en diferentes regiones cerebrales que se ocupan del análisis de la organización melódica y temporal, además del análisis del repertorio. Por lo tanto, es necesaria una gran red neuronal córtico-subcortical ascendente y descendente, entre ambos hemisferios y además conexiones hacia el cerebelo, que permiten la creación de la percepción musical.

Los trastornos de la percepción musical se pueden clasificar a grandes rasgos, en amusia y alucinaciones musicales. La amusia, en términos generales, es la pérdida específica de la percepción musical, que puede ser congénita o adquirida. Un grupo creciente de pacientes con amusia adquirida son la mayoría de los usuarios de implante coclear. Por otro lado, las alucinaciones musicales son descripciones verbales de experiencias sensoriales acústicas de tipo musical que no están explicadas por un estímulo externo y están presentes en pacientes con patología neurológica y psiquiátrica, pero también en personas con sordera profunda bilateral.

Actualmente, existen disponibles algunos instrumentos para evaluar las alteraciones de la percepción musical, siendo la batería de Montreal para la evaluación de amusia, una de las pruebas musicales más utilizadas que permite estimar las habilidades perceptuales y de memoria en adultos y recientemente también en niños. Los trastornos de la percepción musical son patologías emergentes con impacto en la calidad de vida de los pacientes.
\end{abstract}

Palabras clave: Música, amusia, alucinaciones musicales, tono, melodía, ritmo.

\begin{abstract}
Music perception is an experience that involves cognitive and emotional sensations according to the cultural background of the subject. The musical processing begins at the periphery in the auditory system and then in the central level is divided in melodic and temporal processing including the musical repertory. An extensive cortical and subcortical neuronal network is needed for the creation of the musical experience including ascending and descending pathways, both cerebral hemispheres and the cerebellum.

Musical perception disorders can be classified in two main groups; amusia and musical hallucinations. Amusia corresponds to the inability to recognize musical tones that can
\end{abstract}

\footnotetext{
${ }_{1}$ Programa de Magíster en Neurociencias, Facultad de Medicina, Universidad de Chile.

${ }^{2}$ Servicio de Otorrinolaringología, Hospital Clínico Universidad de Chile.

${ }^{3}$ Programa de Fisiología y Biofísica, ICBM, Facultad de Medicina, Universidad de Chile.
} 
be congenital or acquired. A growing group of acquired amusia patients is the majority of cochlear implant users. On the other hand musical hallucinations are the perception of music without an external stimulus, which can be produced by neurologic and psychiatric states, but also in persons with profound bilateral deafness.

Nowadays the Montreal battery of evaluation of amusia is available to evaluate the perceptual skills and memory in adults and child; this test allows the detection of musical perception disorders that are seen as an emerging group of diseases with an important impact in life quality of our patients.

Key words: Music, amusia, musical hallucinations, pitch, melody, rhythm.

\section{INTRODUCCIÓN}

La música es una experiencia perceptual creada a partir de estímulos auditivos y táctiles, que en la mayoría de los seres humanos produce efectos motores, emotivos y cognitivos. Desde el punto de vista auditivo, la percepción musical podría intentar definirse como la percepción de estímulos acústicos organizados coherentemente en diferentes tonos y tiempos, que activan diferentes regiones del cerebro, incluyendo la corteza auditiva y núcleos subcorticales. La música es un tipo de comunicación cuyos estímulos pueden ser memorizados en un léxico musical, y dependiendo de la experiencia generan diferentes respuestas cognitivas y emocionales. Sin embargo, la definición de música depende del contexto cultural e histórico de una sociedad y además de la experiencia individual de cada sujeto. Así, ninguna definición puede ser considerada como absoluta.

El músico francés, Claude Debussy, define a la música como "un total de fuerzas dispersas, expresadas en un proceso sonoro que incluye: el instrumento, el instrumentista, el creador y su obra, un medio propagador y un sistema receptor". Este enunciado le da importancia a la música como un método de comunicación entre seres humanos y propone como requisito importante a las vías auditivas involucradas en la percepción musical'. En los últimos 30 años, ha habido un importante avance en el estudio de las bases neurocientíficas de la percepción musical, dado por la aplicación de técnicas que demuestran la organización funcional del "cerebro musical" como la tomografía por emisión de positrones (PET), la tomografía por emisión de fotón único (SPECT) y la resonancia magnética funcional (RMf), junto con el desarrollo de electroencefalogramas de alta densidad y magnetoencefalograma entre otros ${ }^{2}$.
En este artículo se revisan las bases científicas del procesamiento musical normal y se presentan dos grandes grupos de patologías que alteran la percepción musical: (i) amusia o déficit de la percepción musical y (ii) las alucinaciones musicales. Además se discute la utilidad clínica de la batería de Montreal para la detección de amusia (BMEA).

\section{PROCESAMIENTO MUSICAL NORMAL}

La música, como cualquier estímulo acústico, es detectada a nivel coclear y luego la información es conducida a través de la vía auditiva ascendente hacia la corteza auditiva. La vía auditiva aferente involucra a los núcleos cocleares, complejo olivar superior, lemnisco lateral, colículo inferior y núcleo geniculado medial del tálamo ${ }^{3}$. Desde el tálamo auditivo existen conexiones directas a la amígdala cerebral que están implicadas en respuestas relacionadas a la emoción que produce un estímulo auditivo ${ }^{4}$. La corteza auditiva humana se localiza en el giro temporal superior, en relación a la fisura lateral. Histológicamente, la corteza auditiva primaria $\left(A_{1}\right)$ se ubica en la región medial del giro de Heschl, en la porción anterolateral del mismo plano, mientras que la corteza auditiva secundaria $\left(A_{2}\right)$ se encuentra lateral a $A_{1}$. Posterior al giro de Heschl, se encuentra el planum temporale, el que se describe como la corteza auditiva de asociación, basado en su participación en el procesamiento de estímulos tanto auditivos como de otras modalidades 5 . Es importante destacar que los límites macroscópicos pueden variar con respecto a los límites celulares, lo cual debe ser considerado al momento de evaluar los defectos que producen las lesiones evidenciadas con imágenes ${ }^{6}$.

Peretz y cols en el año $2003^{7}$ propusieron un modelo del procesamiento musical que se divide 
en dos grandes grupos: (i) la organización melódica u organización del tono (pitch en inglés), y (ii) la organización temporal de los estímulos musicales (Figura 1).

El tono (pitch) de un estímulo acústico depende de la frecuencia fundamental (F0), que es el primer múltiplo de frecuencia de un conjunto de armónicos, es decir, si un estímulo posee los armónicos de 100, 200, 300 y $400 \mathrm{~Hz}$, la F0 corresponderá al componente de $100 \mathrm{~Hz}$, y la percepción de este tono será de $100 \mathrm{~Hz}$. El tono de un estímulo musical permite reconocer y ordenar a diversos sonidos en una escala musical, lo cual se conoce como notas musicales.

La capacidad de percibir tonos es un atributo fundamental del cerebro, y de esta manera se sabe que existen estímulos auditivos en los que se ha filtrado artificialmente la frecuencia de F0 y sólo se dejan los armónicos (en el ejemplo, 200, 300 y $400 \mathrm{~Hz}$ ). Sin embargo, desde el punto de vista perceptual, el estímulo es percibido de igual manera como un tono de $100 \mathrm{~Hz}$. Este tipo de paradigma psico-acústico se denomina frecuencia fundamental ausente (missing fundamental) y se sabe que está presente en humanos, primates e incluso en roedores como las chinchillas ${ }^{8-10}$. Más aún, se sabe que existen neuronas que responden de manera específica a la Fo de un estímulo presentado en el paradigma de la frecuencia fundamental ausente ${ }^{8}$.

En el ámbito musical, una secuencia de tonos se utiliza para construir melodías o patrones repetidos en el tiempo; acordes que son una presentación simultánea de más de un tono; y la presentación sincrónica de más de una melodía, mejor conocida como armonía. Todas estas variaciones de las notas musicales producen un aumento de actividad cortical fundamentalmente en las regiones anterior y posterior del giro temporal superior en forma bilateral, con mayor grado en el hemisferio derecho ${ }^{11,12}$, pero también se ha visto aumento de actividad en distintas localizaciones de los lóbulos frontales ${ }^{13-15}$. Esto último, pone de manifiesto que la percepción de un tono musical involucra redes neuronales que incluyen las áreas corticales auditivas y además aquellas adyacentes al giro temporal superior, por lo que el reconocimiento de este componente de la música es compartido con el de otros sonidos como el lenguaje ${ }^{16}$.

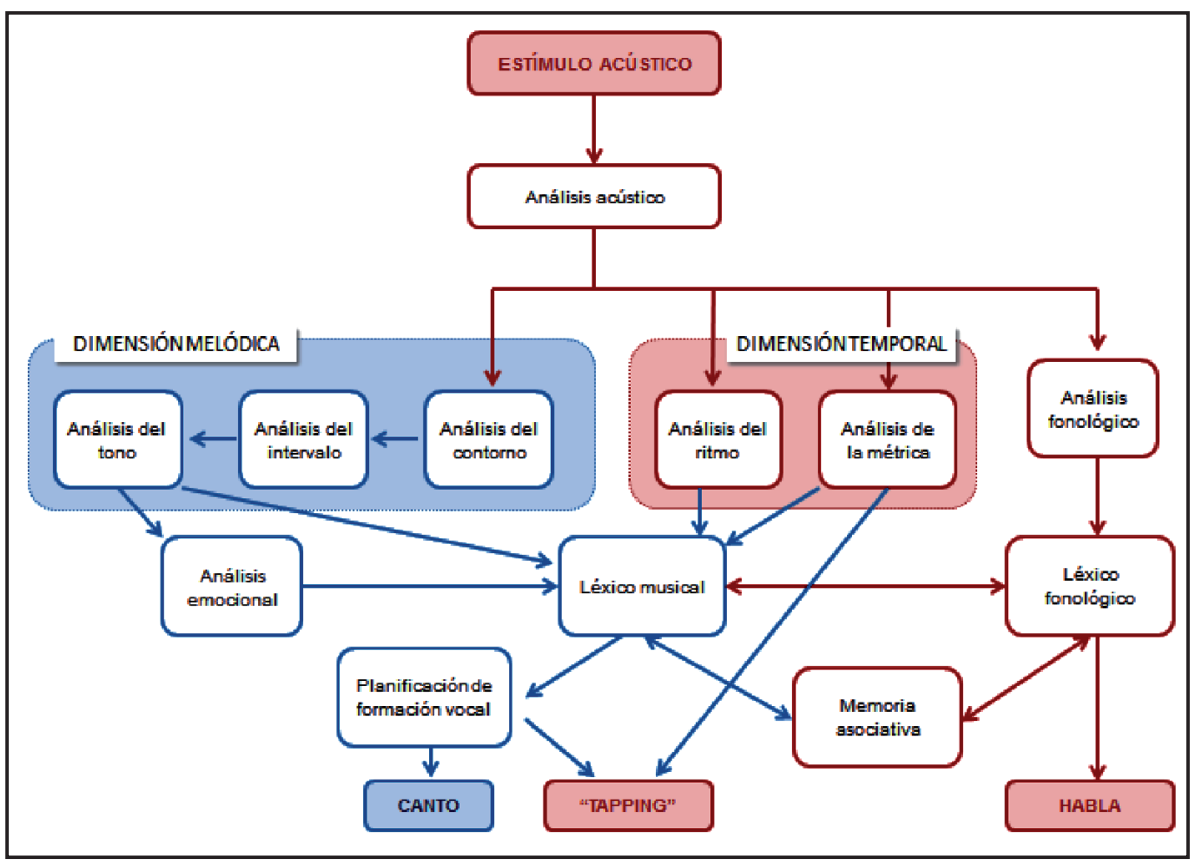

Figura 1. Modelo del procesamiento musical. El procesamiento musical es complejo e involucra numerosas conexiones entre diferentes áreas. El análisis del estímulo musical se divide en una dimensión melódica y una dimensión temporal, pero finalmente ambas confluyen en el repertorio o léxico musical, incluyendo el análisis del lenguaje y de la expresión de emociones. Modificado de Peretz I, Coltheart M. Modularity of music processing. Nature Neuroscience 2003; 6(7): 688-91. 
En cuanto a los sustratos que subyacen al análisis de la organización temporal de la música, es decir, el análisis del ritmo y la métrica, han sido menos estudiados en comparación a la organización tonal. No obstante se ha demostrado la activación de áreas del cerebelo y núcleos de la base, mediante la utilización de RMf y PET ${ }^{17-19}$. De esta forma, se conjetura la teoría motora de la percepción del ritmo y la métrica, dado la activación de áreas del encéfalo que participan en las tareas motoras.

Una vez procesadas las dimensiones melódica y temporal del estímulo musical, el siguiente punto en el modelo es el repertorio. El repertorio o léxico musical, concebido como un sistema de representación perceptual que contiene todas las representaciones de frases musicales específicas a las que un sujeto ha sido expuesto durante su vida, es el punto a partir del cual el análisis del estímulo será direccionado a otras áreas corticales. Estas regiones corresponden a áreas asociativas de memoria y de reconocimiento emotivo. El componente emocional juega un rol primario en la percepción musical, puesto que el estímulo musical es más que el reconocimiento de la melodía y su temporalidad, constituye una experiencia que desencadena una respuesta fisiológica involuntaria e intensa, gatillada por la connotación que cada sujeto le da a cierta clase de música. Incluso, en algunos trabajos se ha evidenciado la activación simultánea, tanto de corteza involucrada en el análisis de la melodía como de estímulos placenteros, vale decir, el cuerpo estriado ventral, la corteza órbito-frontal y la amígdala cerebral ${ }^{20}$.

Por otra parte, se sabe que el sistema eferente auditivo posee vías neuronales que descienden desde la corteza auditiva hacia el colículo inferior y complejo olivar superior y desde aquí, el sistema olivo coclear inerva a las células ciliadas externas de la cóclea ${ }^{21,22}$. Se ha demostrado que la actividad del sistema eferente auditivo, medida como la supresión de la amplitud de emisiones otoacústicas con ruido contralateral, es mayor en músicos que en no músicos, lo que sugiere que el entrenamiento musical incrementa la función eferente auditiva ${ }^{23-26}$. De esta manera Ballaguer y cols $^{27}$ han sugerido que se debe incorporar al sistema eferente auditivo al modelo de procesamiento musical.
Cabe destacar que el modelo sólo está confeccionado para música monofónica, sin letra. El análisis de la lírica de una canción se debería realizar en forma paralela de acuerdo al procesamiento del lenguaje, lo cual muestra que la percepción musical en el cerebro es compleja e involucra numerosas redes neuronales, incluyendo vías aferentes y eferentes (ver Figura 1).

\section{TRASTORNOS DE LA PERCEPCIÓN MUSICAL}

Los trastornos de la percepción musical se pueden clasificar a grandes rasgos en dos grupos: (i) déficit de la percepción musical 0 amusia y (ii) alucinaciones musicales (Tabla 1).

Tabla 1. Clasificación de los trastornos de la percepción musical

\begin{tabular}{|l|}
\hline Amusia \\
\hline Congénita \\
Adquirida \\
- Accidentes cerebrovasculares \\
\hline Implante coclear \\
\hline Alucinaciones musicales \\
\hline Asociadas a sordera \\
Origen neurológico \\
- Romboencefalitis \\
- Epilepsia \\
- Enfermedad de Alzheimer \\
Origen psiquiátrico \\
• Esquizofrenia
\end{tabular}

\section{i. Amusia}

El término amusia se refiere al déficit específico de la percepción musical, sin afectar al componente motor musical (como la producción de voz cantada o ejecución de un instrumento), ni tampoco a los procesos cognitivos o emotivos que subyacen a la percepción musical. De esta manera, este trastorno sensorial específico se puede subdividir en dos tipos: (a) amusia congénita y (b) adquirida ${ }^{28}$.

La amusia congénita fue caracterizada inicialmente por Ayotte y cols en $2002^{29}$, a partir de estudios sistemáticos en sujetos sordos para un tono. Los investigadores enfatizan que este trastorno es una verdadera agnosia sensorial, en la 
cual la percepción de la música es anormal pese a la presencia de audición y cognición conservadas. Aun cuando permanece poco claro, los déficits en la dimensión tonal parecen ser los más variables, mientras que alteraciones en el resto de los dominios no son un hallazgo consistente en los pocos trabajos que existen sobre el tema².

La amusia adquirida, es la que se produce a lo largo de la vida del sujeto, ya sea por un problema a nivel de la vía auditiva aferente 0 a nivel cortical. Son ejemplos usuales de amusia, los pacientes que han padecido un accidente cerebrovascular (ACV), o recientemente, aquellos a los que se les ha instalado un implante coclear $(\mathrm{IC})^{2}$. Históricamente, la amusia adquirida ha sido estudiada en pacientes con lesiones cerebrales, no obstante, esta condición tiene variadas limitaciones inherentes a cualquier estudio neurocognitivo. Entre ellas, el hecho de que los daños cerebrales rara vez son circunscritos, con hallazgos patológicos dictados por la anatomía vascular. Además, el patrón de los déficits cambia constantemente en el transcurso de la enfermedad y el estado premórbido de los pacientes es difícil de considerar, sobre todo por la escasez de síntomas en lo que a detección de alteraciones en la percepción musical se refiere. Por este motivo, la interpretación de funciones particulares puede ser errónea, incluyendo posibles trastornos coexistentes como afasia².

Luego del primer implante coclear en 1957, comenzaron a estudiarse los trastornos de la percepción musical en pacientes que inicialmente tuvieron una experiencia musical y luego perdieron su audición por algún motivo. En ellos se generaría una suerte de amusia adquirida, expresada por el disgusto de las personas que dejan de percibir y disfrutar la música como antes lo hacían ${ }^{30}$.

\section{Amusia adquirida: Implante coclear}

EI IC es un dispositivo electrónico que se introduce en la escala timpánica de la cóclea para estimular eléctricamente al nervio auditivo de pacientes con sordera profunda de origen coclear. Si bien los modelos actuales de IC cuentan con un número cercano a 20 electrodos, se ha visto que para lograr el reconocimiento de melodías complejas con $80 \%$ de respuestas correctas, se requerirían al menos 40 electrodos verdaderos ${ }^{31}$.
Los trastornos de la percepción musical en usuarios de IC han sido estudiados y comparados con distintos grupos de sujetos, utilizando para ello diferentes métodos de detección. Cooper y cols en $2003^{32}$, exploraron la utilidad de la BMEA en pacientes implantados y compararon los resultados con sujetos normales a los que les presentaron los estímulos con un filtro que simulaba un implante. Resultó que ambos grupos mostraron el típico patrón de una alteración de la percepción musical, en el que las puntuaciones son más altas en las pruebas que miden la percepción de los aspectos temporales de la música (ritmo y métrica) versus las pruebas melódicas (escala, contorno e intervalo).

En otros trabajos, se han comparado las dificultades que presentan los pacientes con IC y los usuarios de audífonos, y aquellos pacientes usuarios de implante por una sordera adquirida versus una sordera congénita, en cuanto a su capacidad para percibir estímulos musicales y gusto por la música. Looi y cols en $2008^{33}$, publicaron un estudio basado en la hipótesis de que los usuarios de audífonos se desempeñarían mejor que los portadores de IC en las pruebas que miden la dimensión melódica, pero tendrían una actuación similar en las pruebas de la dimensión temporal. La suposición se comprobó al demostrar que los sujetos con audífonos tenían resultados significativamente mejores en los ensayos melódicos, pero muy similares en aquellos que evaluaban el ritmo. Además de lo anterior, también estudiaron las posibles diferencias entre los grupos en cuanto a su capacidad para identificar instrumentos musicales 0 conjuntos, pero no encontraron ninguna diferencia. Así concluyen que los usuarios de audífonos con una pérdida auditiva en niveles análogos a los pacientes implantados, tienen un mejor rendimiento en las pruebas de percepción musical, pero ambos grupos en gran parte eran incapaces de lograr una percepción precisa 0 efectiva, independiente del dispositivo que usaran. Esto último resulta interesante, debido a que en trabajos que evalúan la satisfacción de la apreciación musical por parte de ambos tipos de sujetos, se ha visto que si bien, ninguno de los dispositivos permite una valoración placentera y que en la medida que suenan más instrumentos es más difícil apreciar el estímulo musical, los 
usuarios de IC juzgan que la música les es más agradable ${ }^{30}$.

En otra investigación, el equipo de trabajo de Looi comparó los resultados del desempeño de usuarios de IC a largo plazo, usuarios de audífonos y pacientes que previamente usaban audífonos y fueron sometidos a una cirugía de implante. Los resultados mostraron el mismo patrón, con un empeoramiento de la respuesta en la organización del pitch, luego de 3 meses de la cirugía. Al final, se concluye que es probable que el uso de ambos dispositivos al mismo tiempo, pudiera proporcionar una puntuación más alta para estos sujetos ${ }^{34}$.

Debido a las limitaciones que tiene el implante coclear para permitir la efectiva y precisa percepción de un estímulo musical, se planteó en algún momento que los niños usuarios de implante desarrollarían una percepción anormal de las señales musicales a nivel cortical. Por ello, Hopyan y cols en $2012^{35}$, diseñaron un estudio que comparó la percepción de música entre niños usuarios de IC y niños con audición normal, tanto en la percepción del tono, como del ritmo y la memoria para recordar piezas musicales, utilizando una versión infantil de la BMEA. Los niños implantados fueron menos exactos que sus pares con audición conservada en sus respuestas, pero mejoraron al discernir los cambios de ritmo y en recordar las piezas musicales. No obstante, al contrario de lo que se hubiese esperado, las habilidades para escuchar señales musicales, mejoraron en la medida que la edad de implante fue más tardía. Este hecho podría ser secundario a que los niños que se implantaron más tarde desarrollaron tal vez, una audición de frecuencias bajas con la ayuda de audífonos, lo cual parece establecer una base para la percepción de música a la que se puede acceder posteriormente con el uso de IC. Estos resultados sugieren que una exposición a experiencias acústicas en forma temprana, mejora la percepción de la música utilizando dispositivos eléctricos, especialmente para la detección de diferencias en el ritmo y la memorización de melodías. A pesar de que los niños que usan IC tuvieron respuestas significativamente más pobres que sus compañeros oyentes en la detección de señales musicales, algunos de ellos fueron capaces de percibir en un rango considerado normal. Estos niños fueron los que estuvieron expuestos a estímulos de baja frecuencia por el uso de audífonos, usaron audífonos por más tiempo y fueron implantados más tardíamente. Esto habla de la importancia de la estimulación acústica para el desarrollo auditivo y sugiere que estas vías de desarrollo pueden adaptarse al nuevo estímulo eléctrico proporcionado por un implante coclear ${ }^{35}$.

La incapacidad para identificar información en la organización del pitch se atribuye principalmente a la resolución de frecuencia del implante. Sin embargo, a pesar de que los niños usuarios de implante rindieron mejor en las pruebas que evalúan ritmo, aun lo hicieron peor que el grupo control. Esto podría explicarse porque en la evaluación del ritmo se incluye la discriminación de la variación tonal; en efecto, cuando estas variaciones de tono son removidas, algunos individuos son capaces de discriminar patrones rítmicos como sujetos normales ${ }^{35}$.

En cuanto a la prueba de memoria de la batería, el equipo de Hopyan encontró que fue la prueba de mejor rendimiento de los niños usuarios de IC, aunque aún están por debajo de los controles. Esto indica que estos niños tenían cierta capacidad para retener melodías, siendo este hallazgo aparentemente exclusivo de los usuarios de IC infantiles, comparados con los adultos. En los adultos se ha demostrado un mal desempeño en la prueba de memoria de la BMEA ${ }^{32}$. El recuerdo del estímulo musical es esencial para que se genere placer a partir del éste; de hecho, las personas habitualmente prefieren las melodías familiares sobre las nuevas. Se sabe que los niños que utilizan IC disfrutan la música, participan en actividades musicales ${ }^{36-38}$ y pueden reconocer canciones familiare ${ }^{39,40}$. Por el contrario, un adulto usuario de IC suele calificar como un sonido molesto a los estímulos musicales y en general prefieren no escuchar música ${ }^{41,42}$.

En suma, y si finalmente se comparan los problemas en la percepción musical, para los pacientes con amusia adquirida, difieren en cierta medida para aquellos sujetos que han padecido un ACV y para los usuarios de IC. Mientras para los primeros se describen alteraciones a nivel de la organización del análisis tonal y del contorno melódico (inflexiones del ritmo) dependiendo del lugar del infarto y del compromiso cognitivo relacionado ${ }^{28}$, para los segundos se encuentra principalmente un defec- 
to a nivel del reconocimiento en la organización tonal ${ }^{32}$.

A nivel central, los defectos en el análisis tonal han sido asociados con daños predominantemente en las áreas auditivas del hemisferio derecho, incluyendo la zona lateral al giro de Heschl, el planum temporale, la unión parieto-temporal y el opérculo frontal2. Por su parte, la alteración del análisis del contorno melódico se debe a una alteración en $A_{2}$. Esto se observa en personas con lesiones cerebrales, pero también como consecuencia de un déficit en la percepción de la organización tonal en los pacientes implantados dado que los sonidos son muy complejos y el estímulo varía con respecto a sujetos que tienen audición normal. Sin embargo, en cuanto al análisis del ritmo, mientras las personas con daño cortical en niveles superiores al giro de Heschl, presentan un déficit, los sujetos con IC parecen no tener problemas ${ }^{2,43}$.

\section{Batería de Montreal para la evaluación de amusia (BMEA)}

El interés del grupo de Peretz en los desórdenes musicales resulta de la observación acerca de que las funciones musicales se pueden deteriorar 0 preservar de un modo altamente selectivo, es decir, un daño cerebral puede interferir con habilidades musicales mientras el resto del sistema cognitivo, incluyendo el lenguaje, permanece esencialmente intacto. No sólo lo anterior, sino que dentro de los componentes de la percepción musical, no todos estarán igualmente dañados ${ }^{28}$.

Por este motivo, desde 1987, este grupo ha desarrollado una batería de pruebas musicales para evaluar las habilidades perceptuales y de memoria en adultos. Este conjunto de ensayos es el que recibe el nombre de BMEA, el cual se ha ido perfeccionando hasta la versión más reciente, publicada en 2003. La BMEA contiene 6 pruebas que permiten la evaluación del funcionamiento de cada componente relevante del modelo de percepción musical propuesto por el grupo de Peretz en 2003 (ver Figura 1). De acuerdo con esto, las pruebas están destinadas a evaluar el contorno melódico, el intervalo tonal, la escala o tono, el ritmo, la métrica y la memoria musical. Cada prueba cuenta con el mismo conjunto extractos de piezas musicales monofónicas, novedosas para los sujetos y que fueron compuestas de acuerdo a la pauta del sistema tonal occidental (con 12 semitonos). El material musical fue intencionalmente escrito con la suficiente complejidad para garantizar su procesamiento como una estructura significante y no sólo como una secuencia simple de tonos. Cada pieza musical dura en promedio 5,1 segundos, excepto las piezas que evalúan la métrica y que tienen una duración promedio del doble ${ }^{28}$.

Esta batería ha sido probada y validada en población normal y es una herramienta muy útil, que tiene una sensibilidad mayor al $80 \%$ en la detección de trastornos de la percepción musical. De hecho, ha mostrado que los pacientes que padecen amusia adquirida por un ACV tienden a presentar problemas en las pruebas que evalúan la organización del pitch y en la prueba que valora los déficits del ritmo, mientras que los pacientes usuarios de implante coclear tienen una alteración a nivel de la organización del pitch únicamente ${ }^{32}$.

\section{ii. Alucinaciones musicales}

Las alucinaciones musicales, han sido reportadas desde distintas perspectivas: auditiva, neurológica y psiquiátrica, en las que la interpretación y descripción del fenómeno deben ser comprendidas desde la causa originaria. No hay estudios disponibles que expongan resultados contundentes, salvo en audición, en los cuales puede evidenciarse un correlato neural con áreas temporales superiores. Algunas de las causas que se mencionan como productoras de alucinaciones musicales y pertenecientes a las categorías descritas son: sordera bilateral profunda, epilepsia, enfermedad de Alzheimer en algunos casos, romboencefalitis y esquizofrenia, siendo todas en general muy complejas de ser evaluadas por las características de los pacientes, al contrario de aquellos que padecen habitualmente una patología causal de amusia adquirida².

Las alucinaciones musicales se definen como la experiencia subjetiva de escuchar música, 0 aspectos de ella, sin estímulos externos presentes $^{44}$. Aún no se ha logrado identificar la etiología de las alucinaciones musicales, pero se ven involucrados tanto procesos periféricos como centrales y se observan en distintos escenarios clínicos así como frente al consumo de fármacos psicoactivos $^{2,45}$. 


\section{Alucinaciones musicales en sordos profundos}

Para entender las alucinaciones musicales en general, pero principalmente en pacientes con patología auditiva, es necesario recordar que el procesamiento melódico de la música ocurre principalmente en las regiones anterior y posterior del giro temporal superior en forma bilateral, con mayor grado en el hemisferio derecho, mientras que el procesamiento rítmico ocurriría en centros motores como núcleos de la base y cerebelo ${ }^{46}$. Goycoolea y cols en $2004^{47}$ llevaron a cabo un estudio para tratar de determinar, entre otras cosas, si existe un sustrato biológico para el proceso de recordar percepciones auditivas previas, y si fuera posible, sugerir un locus para la memoria auditiva, suponiendo que el proceso de memoria auditiva juega un rol de impacto en las alucinaciones de este tipo. Para ello realizaron una evaluación neurofuncional con SPECT de voluntarios sanos sometidos a tonos puros por un oído, y de una paciente con sordera profunda bilateral que en el momento de la medición presentaba la sensación de estar escuchando una melodía conocida sin estímulo externo. Los resultados mostraron una importante activación del área que circunda al giro angular (área 39 de Brodmann) del hemisferio derecho, contralateral al oído estimulado, tanto en sujetos sanos como en la paciente que presentó la alucinación musical y no fue sometida a estimulación auditiva. Además, se vio también la activación de las cortezas frontales ejecutivas en forma bilateral, varias estructuras diencefálicas con predominio del lado derecho y finalmente, se observó una hipoperfusión del lobo temporal del mismo lado. Esto último fue un hallazgo que no se había reportado previamente y los autores piensan que puede corresponder a la presencia de circuitos inhibitorios y no solamente excitatorios que juegan un rol en la vía auditiva, desde el oído interno. El oído interno tendría una función de gatillo inhibitorio o desinhibitorio y no solamente excitatorio, lo cual podría relacionarse con el fenómeno de las alucinaciones auditivas evidenciado en la paciente con sordera profunda bilateral, que tuvo el mismo patrón de activación que los sujetos normales. En otro trabajo de los mismos autores ${ }^{48}$, se amplió la muestra de pacientes sordos y se encontraron los mismos hallazgos, usando la misma técnica de imagen funcional. Se encontró una mayor activación del área 39 de
Brodmann, por lo cual se concluyó que ésta podría estar asociada al centro de la memoria auditiva, siendo de especial relevancia en las alucinaciones musicales. Goycoolea y su equipo proponen que en los pacientes con sordera profunda bilateral existe una desinhibición neuronal en el área de la memoria auditiva, lo que produce la imposibilidad de mantener almacenada las sensaciones musicales, generando así una liberación perceptual ${ }^{48,49}$.

Por otra parte, existen varios trabajos en los cuales se describen alucinaciones musicales en pacientes con sordera profunda bilateral, desde una perspectiva más clínica. En el trabajo de Griffiths del año $2000^{46}$, se mencionan 6 sujetos en los que las alucinaciones aparecieron tanto de manera abrupta como paulatina. Una vez adquirida la sordera profunda bilateral, éstas variaban desde la presencia de tinnitus hasta la percepción completa de melodías familiares para cada uno de los sujetos, como himnos y/o canciones populares que podían a su vez manifestarse solo como melodías o con la presencia de letra incluida ${ }^{46,50}$. Todos los pacientes relatan las alucinaciones como percepciones idénticas de las canciones que oían previo a la aparición de la sordera. Stewart y cols ${ }^{2}$ describen que las alucinaciones musicales se producen principalmente en pacientes de mediana edad 0 adultos mayores con hipoacusia severa a hipoacusia profunda bilateral, existiendo mayor prevalencia en el sexo femenino. Por otra parte, la aparición de alucinaciones no se asocia a sentimientos de estrés y/o emociones de connotación negativa².

\section{Alucinaciones musicales en otras patologías}

En los pacientes con algún tipo de lesión neurológica central, el origen de las alucinaciones es variada ya que puede ocurrir en múltiples regiones involucradas en el procesamiento musical. Se pueden observar en lesiones de tronco cerebral, accidentes vasculares en lóbulo temporal, principalmente derecho, epilepsia, aneurismas cerebrales, tumores y algunos tipos de demencias, entre otros. Existen múltiples casos descritos en la literatura y cada presentación varía según la localización de la lesión $n^{2,45,51}$.

En relación a las patologías psiquiátricas, las alucinaciones musicales se ven con más frecuencia en trastornos depresivos, esquizofrenia y trastorno obsesivo compulsivo. A nivel cortical los procesos 
que subyacen a las alucinaciones son mucho más complejos. Si bien se ha visto que se activan las áreas corticales anteriormente mencionadas, hay participación importante del sistema límbico y de áreas involucradas en memoria de trabajo y lenguaje, sobre todo en esquizofrenia ${ }^{2,52}$.

Por último, múltiples fármacos pueden provocar alucinaciones musicales, en especial el grupo de Ios opioides, salicilatos y benzodiacepinas. La gran mayoría de los pacientes que consumen estos medicamentos tienen patologías psiquiátricas asociadas, por lo que sería un factor de riesgo agregado. En el caso de los opioides la producción de alucinaciones musicales sería a través de mecanismos periféricos y centrales, esto en base a la presencia de receptores opioides en la cóclea, además de los efectos centrales de estos fármacos ${ }^{45,53}$.

En relación al tratamiento de las alucinaciones, éste debe estar enfocado a la posible causa. Es importante destacar que todas las categorías en que se presentan las alucinaciones musicales pueden estar asociadas, lo cual transforma al tratamiento en un difícil desafío. Se ha visto que el uso de audífonos es de utilidad ${ }^{50}$, al aumentar el estímulo externo y amplificar el sonido, pero sólo en las alucinaciones relacionadas con pérdidas auditivas. Respecto a tratamientos farmacológicos, como ya se mencionó, deben estar enfocados en la patología específica y las diferentes series internacionales no han llegado a resultados consistentes ${ }^{45}$.

\section{CONCLUSIONES}

Los trastornos de la percepción musical son patologías emergentes y tienen un rol muy importante en la calidad de vida de los pacientes, pero aún se desconoce gran parte de su fisiopatología. Pese a ello, el desarrollo de pruebas de evaluación de la percepción musical permitirá clasificar los diferentes tipos de trastornos de la percepción musical presentes en pacientes con accidentes cerebro-vasculares y usuarios de implantes cocleares 0 audífonos.

\section{BIBLIOGRAFÍA}

1. Grout D, Palisca CV. Historia de la música occidental: la música europea desde la década de 1870 hasta la Primera Guerra Mundial. Alianza Música. $3^{a}$ Edición. Madrid: Alianza Editorial 2001: 845-94.

2. Stewart L, von Kriegstein K, Warren J, Griffithst. Music and the brain: disorders of musical listening. Brain 2006; 129: 2533-53.

3. Délano, PH. Central Auditory System. Atlas of Otologic Surgery and Magic Otology, Volume 1. Pages 77-84. Jaypee Brothers. Ed. MV Goycoolea, 2012.

4. LeDoux JE, Sakaguchi A, Reis D. Subcortical efferent projections of the medial geniculate nucleus mediate emotional responses conditioned to acoustic stimuli. J Neurosci 1984; 4: 683-98.

5. GrifFiths TD, Warren JD. The planum temporale as a computational hub. Trends Neurosci 2002; 25: 348-53.

6. Rademacher J, Morosan P, Schormann T y col. Probabilistic mapping and volume measurement of human primary auditory cortex. Neuroimage 2001; 13: 669-83.

7. Peretz I, Coltheart M. Modularity of music processing. Nature Neuroscience 2003; 6(7): 688-91.

8. Bendor D, Wang $X$. The neuronal representation of pitch in primate auditory cortex. Nature 2005; 436: 1161-5.

9. Bendor D, Wang $X$. Cortical representations of pitch in monkeys and humans. Curr Opin Neurobiol 2006; 16: 391-9.

10. SHofner WP. Perception of the missing fundamental by chinchillas in the presence of low-pass masking noise. J Assoc Res Otolaryngol 2011; 12: 101-12.

11. Griffiths TD, Buchel C, Frackowiak RSJ, Patterson $\mathrm{RD}$. Analysis of temporal structure in sound by the human brain. Nat Neurosci 1998; 1: 422-7.

12. Patterson RD, Uppenkamp $S$, Johnsrude IS, Grifiths TD. The processing of temporal pitch and melody information in auditory cortex. Neuron 2002; 36: 767-76.

13. Zatorre RJ, Evans AC, Meyer E. Neural mechanisms underlying melodic perception and memory for pitch. J Neurosci 1994; 14: 1908-19.

14. Koelsch S, Siebel WA. Towards a neural basis of music perception. Trends Cogn Sci 2005; 9: 578-84.

15. Levitin DJ, Menon V. Musical structure is processed in 'language' areas of the brain: a 
possible role for Brodmann area 47 in temporal coherence. Neuroimage 2003; 20: 2142-52.

16. Price C, ThierRy G, Griffiths T. Speech-specific auditory processing: where is it? Trends Cogn Sci 2005; 9: 271-6.

17. Penhune VB, Zatorre RJ, Evans AC. Cerebellar contributions to motor timing: a PET study of auditory and visual rhythm reproduction. J Cogn Neurosci 1998; 10: 752-65.

18. Sakai K, Hikosaka 0, Miyauchi S, Takino R y col. Neural representation of a rhythm depends on its interval ratio. J Neurosci 1999; 19: 10074-81.

19. Xu D, Liu T, Ashe J, Bushara KO. Role of the OlivoCerebellar System in Timing. J Neurosci 2006; 26: 5990-5.

20. Blood AJ, Zatorre R. Intensely pleasurable responses to music correlate with activity in brain regions implicated in reward and emotion. Proc Natl Acad Sci USA 2001; 98: 11818-23.

21. Délano PH, Robles I, Robles L. Sistema eferente auditivo. Rev Otorrinolaringol Cir Cabeza Cuello 2005; 65: 55-62.

22. Robles L, Délano PH. Efferent system. The senses: a comprehensive reference. 2008. London: Academic Press. pp. 413-45.

23. Micheyl C, Carbonnel 0, Collet L. Medial olivocochlear system and loudness adaptation: differences between musicians and nonmusicians. Brain Cogn 1995; 29(2): 127-36.

24. Micheyl C, Khalfa S, Perrot X, Collet L. Difference in cochlear efferent activity between musicians and non-musicians. Neuroreport 1997; 8(4): 1047-50.

25. Perrot X, Micheyl C, Khalfa S, Collet L. Stronger bilateral efferent influences on cochlear biomechanical activity in musicians than in non-musicians. Neurosci Lett 1999; 262(3): 167-70.

26. Brashears SM, Morlet TG, Berlin Cl, Hood LJ. Olivocochlear efferent suppression in classical musicians. J Am Acad Audiol 2003; 14(6): 31424.

27. Balaguer-Ballester E, Clark NR, Coath M, Krumbholz K, Denham SL. Understanding pitch perception as a hierarchical process with topdown modulation. PLoS Comput Biol 2009; 5(3): e1000301.

28. Peretz I, Champod A-S, Hyde KL. Varieties of musical disorders. The Montreal battery of evaluation of amusia. Ann NY Acad Sci 2003; 999: 58-75.

29. Ayotte J, Peretz I, Hyde K. Congenital amusia. A group study of adults afflicted with a musicspecific disorder. Brain 2002; 125: 238-51.

30. Lool V, McDermott H, McKay C; Hickson L. Comparisons of Quality Ratings for Music by Cochlear Implant and Hearing Aid Users. Ear \& Hearing 2007; (28): 59-61.

31. Galvin III J, Fu Q, Shannon R. Melodic Contour Identification and Music Perception by Cochlear Implant Users. Annals of the New York Academy of Sciences 2009; 1169: 518133.

32. Cooper W, Tobey E, Loizou P. Music Perception by Cochlear Implant and Normal Hearing Listeners as Measured by the Montreal Battery for Evaluation of Amusia. Ear Hear 2008; 29(4): 618-26.

33. Lool V, McDermott H, McKay C, Hickson L. Music Perception of Cochlear Implant Users Compared with that of Hearing Aid Users. Ear \& Hearing 2008; 29: 421-34.

34. Looi V, McDermott H, McKay C, Hickson L. The effect of cochlear implantation on music perception by adults with usable pre-operative acoustic hearing. International Journal of Audiology 2008; 47: 257-68.

35. Hopyan T, Peretz I, Chan LP, Papsin BC, Gordon Ka. Children Using Cochlear Implants Capitalize on Acoustical Hearing for Music Perception. Front Psychol 2012; 3: 425.

36. Gfeller K, Witt SA, Spencer LJ, Stordahl J, Tomblin B. Musical involvment and enjoyment of children who use cochlear implants. Volta Rev 1999; 100: 213-33.

37. StORDAHL J. Song recognition and appraisal: a comparison of children who use cochlear implants and normally hearing children. J Music Ther 2002; 39(1): 2-19.

38. Nakata T, Trehub SE, Mitani C, Kanda Y, Shibasaki A, Schellenberg EG. Music recognition by Japanese children with cochlear implants. J Physiol Anthropol Appl Human Sci2005; 24(1): 29-32.

39. Vongpaisal T, Trehub SE, Schellenberg EG. Song recognition by children and adolescents with cochlear implants. J Speech Lang Hear Res 2006; 49(5): 1091-103. 
40. Trehub SE, Vongpaisal T, Nakata T. Music in the lives of deaf children with cochlear implants. Ann N Y Acad Sci 2009; 1169: 534-42.

41. Gfeller K, Christ A, Knutson JF, Witt S, Murray KT, TYLER RS. Musical Backgrounds, listening habits, and aesthetic enjoyment of adult cochlear implant recipients. J Am Acad Audiol 2000; 11: 390-406.

42. Leal MC, Shin YJ, Laborde ML y col. Music perception in adult cochlear implant recipients. Acta Otolaryngol 2003; 123(7): 826-35.

43. McDeRMotT HJ. Music perception with cochlear implants: a review. Trends Amplif 2004; 8(2): 49-82.

44. Berríos GE. Musical hallucinations. A historical and clinical study. Br J Psychiatry 1990; 156: 188-94.

45. Cope TE, Baguley DM. Is musical hallucination an otological phenomenon? A review of the literature. Clin Otolaryngol 2009; 34(5): 423-30.

46. GRIFFITHS TD. Musical hallucinosis in acquired deafness. Phenomenology and brain substrate. Brain 2000; 123: 2065-76.

47. Goycoolea M, Mena I, Neubauer S. Estudios funcionales de la corteza auditiva humana, de la memoria auditiva y alucinaciones auditivas. Alasbimn Journal 2004; 6 (25). Article N AJ25-1.
48. Goycoolea M, Mena I, Neubauer S. Spontaneous musical auditory perceptions in patients who develop abrupt bilateral sensorineural hearing loss. An uninhibition syndrome? Acta Otolaryngol 2006; 126: 368-74.

49. Evers S, Ellger T. The clinical spectrum of musical hallucinations. J Neurol Sci 2004; 227(1): 55-65.

50. Sánchez TG, Rocha SC, Knobel KA, Kı MA, Santos RM, Pereira CB. Musical hallucination associated with hearing loss. Arq Neuropsiquiatr 2011; 69(2B): 395-400.

51. Calabro R, Baglieri A, Ferlazzo E, Passari S, Marino S, Bramanti P. Neurofunctional assessment in a stroke patient with musical hallucinations. Neurocase: The Neural Basis of Cognition 2012. DOI:10.1080/13554794.2011 .633530 .

52. Daalman K, Boks MP, Diederen KM y col. The same or different? A phenomenological comparison of auditory verbal hallucinations in healthy and psychotic individuals. J Clin Psychiatry 2011; 72: $320-5$

53. Jongkamonwiwat N, Phansuman-Pujito P, Sarapoke $P$ y coL. The presence of opioid receptors in rat inner ear. Hear Res 2003; 181: 85-93. 